\title{
Internacionalización del territorio antioqueño: Una lección por aprender ${ }^{*}$
}

\author{
Jahir Alexánder Gutiérrez-Ossa, J Jefferson Carmona² \\ Institución Universitaria Tecnológico de Antioquia (Colombia)
}

Recibido: agosto 27 de 2014 - Revisado: noviembre 9 de 2014 - Aceptado: diciembre 12 de 2014

Referencia formato APA: Gutiérrez-Ossa, J. A., \& Carmona, J. (2014). Internacionalización del territorio Antioqueño: Una lección por aprender. Revista Cientifica Guillermo de Ockham, 12(2), 85-93.

\section{Resumen}

El artículo analiza los aspectos, elementos e indicadores que revisten los procesos de internacionalización trazados hasta ahora en el departamento de Antioquia, vinculados a dispersos intereses, sin una apuesta clara. La internacionalización obedece a disímiles interpretaciones, que difícilmente, pueden ser replicables simultáneamente. De allíla relevancia de plantear el escenario que se requiere para fundar las bases de un proceso idóneo y estructurado que permita elaborar un marco sistémico de incumbencia general de todos en esta problemática. Si bien, en términos generales, ha sido dispuesta la normatividad constitucional y central referida a lo internacional, el ejercicio aplicado por los departamentos y municipios es exiguo, con excepción de los departamentos de Antioquia y Cundinamarca. Igualmente, la perspectiva territorial desempeña un papel de cardinal importancia para que los procesos de internacionalización sean cercanos a la programación política y pública como variable de gestión institucional departamental. Antioquia, particularmente, debe establecer una política pública abierta y clara al respecto.

Palabras clave: Acuerdos, internacionalización, territorios, políticas, economía

\section{Internationalization of the Antioquia territory: A lesson to be learned}

\section{Abstract}

The paper analyzes aspects, elements and indicators of the internationalization processes layouts so far in the Department of Antioquia, linked to scattered interest, without a clear commitment. Internationalization is due to dissimilar interpretations, which can hardly be replicable simultaneously, from there, the relevance of raise the scenario that is required to establish the foundations of a sound and structured process to develop a systemic framework of general responsibility of all in the subject. While in general terms, central and constitutional regulations concerning the International has been prepared, the exercise carried out by the departments and municipalities is meager, with

* Este artículo es producto del proyecto de investigación Evaluación geoestratégica del departamento del Chocó en el marco de las trampas del subdesarrollo: el g-8 aid para el departamento del Chocó, financiado por la Dirección de Investigaciones de la Institución Universitaria Tecnológico de Antioquia, ejecutado por el grupo de investigación Observatorio Público (opción TdeA) y registrado en Colciencias durante el periodo de septiembre de 2014 a septiembre 2015.

1. Doctor en Administración Pública de la Atlantic International University (AIU), Honolulú (USA). Magíster en Desarrollo, de la Universidad Pontificia Bolivariana de Medellín. Economista de la Universidad de Medellín, Colombia. Estudios en Especialización de Sistemas de Información Geográfica en la Universidad San Buenaventura Medellín. E-mail: jagogutierrez@gmail.com

2. Administrador público de la Escuela Superior de Administración Pública (APT) Territorial Antioquia-Chocó. Diplomatura en Políticas Públicas con enfoque en Derechos Humanos del Ministerio del Interior y Justicia (ESAP). Miembro de grupo de investigación Observatorio Público, registrado en Colciencias. Institución Universitaria Tecnológico de Antioquia. E-mail: jefersoncm8@gmail.com 
the exception of Antioquia and Cundinamarca. Also, the territorial perspective plays categorically; to make internationalization processes closer to the political and public programming as departmental institutional management variable. Antioquia must in particular, establish an open and clear public policy thereon.

Keywords: Agreements, internationalization, territories, politics, economics

\section{Introducción}

En la estructura de la gestión gubernamental de la administración pública departamental de Antioquia, ha venido ganando terreno la internacionalización desde la perspectiva territorial. Sin embargo, no ha sido definido el espacio de trabajo para diseñar formalmente esquemas y pautas para abordar dicho proceso y solo se han desarrollado aquellos de orden estamental e institucional, como respuesta a la apertura y liberación económica afincada en la desregulación y flexibilización.

El estado actual de referencia, al que responde tradicionalmente el departamento cuando se trata de llevar procesos idóneos y novedosos en materia de gestión gubernamental, ha incidido a través de diversos escenarios para que la internacionalización tenga fundamento en la administración pública departamental y municipal y puedan revestirse las agendas trasversales de internacionalización por parte de los entes territoriales que requieren internalizar dicho proceso en la gestión local.

En el departamento de Antioquia se creó recientemente la red antioqueńa de cooperación internacional, compuesta por diferentes estamentos e instituciones del orden regional y local, entre las que se encuentran la Agencia de Cooperación Internacional de Medellín y la Subsecretaria de cooperación internacional -dependencia del Instituto para el Desarrollo de Antioquia-, que están al frente de los esfuerzos y procesos inherentes a la internacionalización departamental.

La internacionalización aún simboliza el escenario sobre el que trasiegan las relaciones de los países a través de los Estados, a pesar de la extensa literatura y las propuestas que invitan a reconsiderar el liderazgo de los entes territoriales. Asimismo, la interacción sobre la cual es plasmada la internacionalización, continúa al amparo del relacionamiento, cuyo carácter solo responde al interés puntual y mediático creado a través del vínculo, pero con poca trascendencia institucional.

La internacionalización no es la panacea para las administraciones públicas territoriales, pero sí ofrece la posibilidad de apropiar los elementos, las fuentes y los instrumentos de la gestión pública, gracias a la diversidad de áreas que comprende y que con una directa correlación y trabajo, pueden impactar las actividades institucionales abrigadas en los entes territoriales y establecer un marco de referencia que contribuya a la evaluación de la dirección tomada en la administración.

En virtud de lo hasta ahora estimado y para efectos de consideración, la ponencia abarca la siguiente estructura de análisis: en primer lugar, una revisión del contexto y panorama de los procesos de internacionalización ilustrado desde diversas consideraciones. En segundo lugar, establece el estado del arte y de aprendizaje que podría atender el departamento de Antioquia. Y en tercer lugar, la influencia que traería para el departamento la acción de involucrar la internacionalización como referencia de la gestión pública regional.

\section{Contexto e implicación general de la internacionalización}

La internacionalización parte de la identidad nacional y el reconocimiento estatal que cubren y proveen las capacidades necesarias para que cualquier Estado, en cabeza del Gobierno, emprenda actividades, ejercicios y nexos con sus homólogos, reiterando con ello el interés de pasar de un proceso alineado por las circunstancias del conflicto o las disputas, hacia uno de orden proactivo y extenso para el fortalecimiento estamental e institucional de las relaciones a las que hubiere lugar.

El éxito de estos países obedeció a una combinación de factores internos y externos, vinculados en su mayoría a la situación particular de cada uno de ellos. No obstante, un factor común, a saber, la actitud proactiva del gobierno, fomentó el desarrollo mediante la implementación eficaz de una estrategia nacional de transformación productiva a mediano y largo plazo, orientada a la inserción internacional. Dicha estrategia se organizó en el marco de una alianza público-privada (Devlin \& Moguillansky, 2009, p. 99).

El realismo político ha sido amplio dominador de los conceptos y aprehensiones tomadas con respecto a la categorización y evaluación de las relaciones internacionales entre los países, que si bien sirvieron para indicar aliados de contrarios, no hizo más bien a la humanidad que el de cosificar y mediatizar entre los Estados un permanente marco de alteración del orden internacional, expresado en las múltiples contiendas que acompañaron dicho pe-

$86<$ Universidad de San Buenaventura, Cali - Colombia 
riplo, cimentadas por un creciente y sistemático patrón de desconfianza.

Al dejar de lado la idea belicista y guerrerista de las relaciones internacionales, se han definido y habilitado otros escenarios con el propósito de remarcar el estado de entendimiento y reciprocidad de las relaciones internacionales, sin el característico tono pendenciero. Así, las relaciones internacionales pasaron a formar parte de los asuntos de interés y de preocupación de los países, gracias a la entrada de organismos rectores multilaterales en los cuales las relaciones tendrían asiento y eco.

Internacional significa entre naciones, aunque usualmente se entiende como entre Estados, confundiendo así el término con el de interestatal. Hoy, el concepto internacional hace referencia al sistema compuesto por una pluralidad de actores que incluye a los Estados y alude al concepto de relaciones internacionales, todo ello verificado en el ámbito de la evolución de los sistemas internacionales de la modernidad: unilateralismo, alianzas, bilateralismo, multilateralismo bipolar y multilateralismo unipolar. En tal óptica, los dos grandes traumas del siglo pasado (guerras mundiales) marcaron el ritmo y la calidad de la evolución de las instituciones políticas orientadas hacia la constitución de una comunidad internacional (Agudelo, 2011, pp. 16-17).

El desarrollo económico y la elaboración de un marco de trabajo para preservar la paz mundial, fueron considerados como la base fundamental para dar rienda a las relaciones internacionales. Aunque lo ideológico continuó incidiendo en las relaciones, las perspectivas trazadas derivaron en espacios que las revitalizaron en otros aspectos. Contrario a ello, aparecieron una serie de estamentos e instituciones multilaterales tras las cuales se entendería dicho trabajo como una acción mancomunada de todas las naciones.

Esta adhesión indujo la suscripción de diversos acuerdos en múltiples áreas y campos que priorizaron aspectos por encima del incipiente proceso de intercambio y facilitaron la cooperación internacional. La integración económica promovida por los recién creados organismos multilaterales, hizo de mecanismo ordenador de las nacientes propuestas, que a la par con las virtudes de la integración, empezarían a encauzar las relaciones internacionales.

El patrón característico de la cooperación se basó en la integración y proyección de objetivos y propósitos plenos entre los países que acogieron dicha tendencia, como elemento relevante de su accionar internacional. Tal es así, que parte del contexto de la cooperación estuvo consignado en la política estatal de algunas naciones y claro está, de diversos estamentos de carácter público, lo que dio origen a las agencias de cooperación internacional y a las oficinas de relaciones internacionales centrales y descentralizadas de orden territorial.

La educación, a la par con la relación estamental entre los Estados y sus respectivas instituciones, tomaría la delantera del proceso que identificó el sendero económico como cúspide de las relaciones a través de la firma de acuerdos comerciales y de explotación de diversas condiciones, en los que los países desempeñarían el papel de puente para que, en consecuencia, el campo económico se ordenara como bastión de la regulación de las relaciones internacionales.

En otras tradiciones, se le toma como mero descriptor o dato, entendiendo la globalización como la dimensión internacional del desarrollo o de la evolución urbana reciente. Más que explorar si las ciudades examinadas convergen con toda la categoría de ciudad-global o no, se preocupan por cuantificar los grados y tipos de internacionalización de la vida urbana, especialmente en lo funcional y económico (Cuervo, 2003, p. 17).

El protagonismo logrado por la tendencia económica en cuanto a la materialización de las relaciones internacionales, comenzaría a segregar las ideas resguardas en las escuelas de pensamiento de las relaciones internacionales, arraigadas en el realismo de los aliados y contrarias a la posición conductual o comportamental de las relaciones provista por el behaviorismo, que terminarían por ser explicadas desde el neorrealismo activo, mas no paladino, a la vez que por un posbehaviorismo poco ilustrado.

La reconversión sobre las que terminarían enfiladas las posiciones más duras de las escuelas de las relaciones internacionales -aún en medio de los cambios y el aparente estado anímico de transformación-no posibilitarían la entrada de otros escenarios que si bien estaban a la par en cuanto al interés de las relaciones -por ejemplo, en el caso de la cooperación internacional- eran observadas con amplio recelo debido al poco conocimiento de las interacciones derivadas de ella y sus alcances.

En el mundo de la globalización las experiencias de Cooperación Internacional al Desarrollo aportan una nueva savia a la realidad conflictiva en la que estamos inmersos. Es notorio que la globalización como proceso se caracteriza por la internacionalización de la economía, la política y la cultura con tal vertiginosidad y complejidad que sólo es comparable con la revolución industrial (Márquez y Galindo Pérez de Azpillaga, 2007, p. 205).

A la par con la cuenta pasada a las relaciones internacionales, así mismo fueron revisados el contenido y los logros 
de la cooperación internacional, toda vez que las razones sobre las cuales había sido fundada a la luz de la ayuda, la financiación y la transferencia de recursos entre los Estados, no había logrado su propósito, hecho que restó carácter al objetivo de superar problemáticas asociadas a la pobreza -entre otros- prescripción que contribuyó para su replanteamiento.

La relación entre internacionalización y desarrollo territorial es correlativa. En la mayoría de los casos, al menos en los nacionales, se insertó la dimensión internacional como un componente de los planes de desarrollo ya como un objetivo, estrategia o programa. A pesar de lo anterior, falta una visión más integral del desarrollo unida a las estrategias de internacionalización, pues muchas veces el énfasis está en obtener recursos, atraer inversión o aumentar las exportaciones, descuidando otros aspectos tales como la dimensión cultural o la ambiental (Jiménez, Ochoa \& Pineda, 2010, p. 127).

En adelante, la cooperación internacional pasaría a contemplarse desde una perspectiva descentralizada, debido al poco acierto obtenido por cuenta de las vinculaciones y resultados acaecidos bajo la relación directa de los Estados. De ahí que se haya determinado que la cooperación internacional es el nuevo escenario de vinculación de las relaciones internacionales, que acogen a los territorios como protagonistas de primera línea para llevarla a cabo, con dirección y supervisión del orden estamental central y descentralizado.

En el caso de las experiencias internacionales, se concluye que la postura de algunos actores involucrados en las modalidades de internacionalización es deformada, pues aunque en algunos países de América Latina se han realizado avances alrededor del tema, son muchos los gobiernos centrales que aún persisten, casi y de manera exclusiva, en el manejo de dichos procesos. Esto se debe en gran parte a la carencia de mecanismos jurídicos que permitan a los gobiernos territoriales desarrollar este tipo de prácticas y de los enfoques que manejan algunos organismos internacionales frente a la forma y tipo de cooperación que suministran a dichas entidades (Jiménez, Ochoa \& Pineda, 2010, p. 127).

En esencia, las relaciones internacionales pasan tal vez por su mejor momento gracias al protagonismo que les han dado a las regiones y territorios en el mundo, para que lideren por cuenta de sus propios intereses el marco de referencias sobre el cual hilvanar el carácter internacional que requieren sus poblaciones, superando con ello las circunstancias de ensayo-error orquestadas por el patrón ultraeconómico que todavía domina el discurso y la interpretación de las relaciones internacionales, pero que esta vez pone a las regiones en el centro del debate y las hace líderes de su propio proceso de internacionalización.
La apuesta por la internacionalización desde local requiere el análisis detenido de las condiciones del territorio: generadas como estrategia "de abajo hacia arriba"; y que corresponden a las infraestructuras económicas fuertes que respalden las iniciativas propuestas de desarrollo local por parte de las empresas con respaldo del Gobierno; el conocimiento generado por procesos de aprendizaje individuales totalmente racionales que sumen el conocimiento colectivo del territorio, y la flexibilidad arraigada al territorio como propuesta dinámica y conducente a aceptar las condiciones volátiles actuales para el crecimiento. Además de las políticas de internacionalización, generadas como la estrategia de "arriba hacia abajo", impulsando el compromiso por los actores gubernamentales del país al desarrollar políticas reguladoras de los impactos que generan los procesos globalizadores de las economías y logrando el compromiso por parte de los empresarios con elementos de modernización y procesos de adaptación ante las nuevas exigencias productivas, que permitan el logro colectivo del incremento de la competitividad en los mercados (Osorio, 2010, p. 88).

Las relaciones internacionales han tomado el cariz de elemento regulador no solo de la interacción de los países, sino también de la capacidad individual y colectiva que elaboran para promover tareas conjuntas en distintos campos, sobre los cuales la atención no se centra en la observancia de la relación como concurso o puente institucional, sino en los objetivos y propósitos trazados para que sean cumplidas las metas o proyecciones propuestas. La internacionalización forma parte del diálogo de saberes institucionales y se concreta con los intereses interinstitucionales.

\section{Experiencias y lineamientos de la internacionalización para la gestión departamental en Antioquia}

Pese a que Colombia es catalogado como país centralista, las características que denotan el comportamiento de las naciones hacen discrepar de tal aseveración, dado que a nivel institucional, las estructuras de base tipo regional y local han primado en las decisiones económicas, políticas y sociales, amparadas en el esquema constitucional de la descentralización en el que se asienta la internacionalización. No obstante, la aproximación al tema no alcanza a ser contemplada plenamente.

Mientras que los efectos de la globalización y la supra nacionalización en la soberanía del Estado-nación ha sido objeto de una abundante literatura, el otro lado de la moneda -la génesis y las implicaciones de la emergencia del poder subnacional- ha recibido mucho menos atención. No obstante, existe una creciente evidencia de que los cambios en el nivel 
regional y local son tan significativos como aquellos que están ocurriendo en el plano supranacional: [...] el avance imparable de los procesos de descentralización política y fiscal a escala prácticamente universal y la emergencia de nuevas formas de gobierno y organización institucional ancladas en lo local (local governance). En este sentido, algunos analistas llegan a proponer que se está operando una transición desde el Estado-nación hacia el Estado-región (Moncayo, 2004, p. 17).

La realidad es que no existe una formula clara que les indique a las regiones cómo internacionalizarse, pero el primer paso estriba en el propósito de alcanzar esa internacionalización. Sobre esa base, dicha plataforma puede descansar en un perfil político, económico, social, ambiental, que complemente estos elementos para que la internacionalización se produzca como un proceso completo y no imaginario ni marginal.

De allí que pueda hablarse de la globalización como una desterritorialización, como un paso de lo concreto (los territorios vividos, apropiados por sociedades singulares) a lo abstracto (el espacio global de los flujos, de la simultaneidad de lo discontinuo). A medida que el capital y la cultura se globalizan y se multiplica la movilidad de la población, la propia sustancia de los territorios (su economía, su identidad, su gente) parece volverse volátil, indefinida e incierta (Bervejillo, 1994, p. 13).

La internacionalización es un proceso relacional y de redes en el cual la cooperación, las alianzas, el entendimiento y la reciprocidad entre diferentes frentes es evidente. Sin embargo, entenderla como un proceso económico es llevarla a una posición reduccionista, soslayarla con referencia al campo social y pensarla como un problema de sujetos.

Al describir las experiencias colombianas se observó que algunas entidades territoriales (grandes ciudades, algunos departamentos) han podido vincularse a los procesos globales implícitos en la internacionalización, siendo esto posible por la participación en redes, hermanamientos, asociaciones o, en general, con algún tipo de cooperación internacional (tradicional o descentralizada). Ello les ha permitido diseñar estrategias innovadoras en procura de optimizar su gestión pública, estrechar lazos comerciales que mejoren sus economías y la participación activa en escenarios, anteriormente limitados para este tipo de entidades, donde se discuten temas que intervienen directamente con su desarrollo local (Jiménez, Ochoa \& Pineda, 2010, p. 127).

Se intenta que Antioquia pase de ser una mera ubicación geográfica, para transformarse y adaptarse a los cambios y procesos de la internacionalización. Sin dejar de reconocer la validez de dichas directrices a la hora de tomarlas como elementos explicativos de la internacionalización de los territorios, la evidencia real enseña que más allá de ello los territorios presentan otras situaciones que posibilitan su incursión en procesos de internacionalización, dentro de las cuales se encuentran factores axiológicos, sicológicos y sociales, sobre la idea territorial que se pretende internacionalizar.

Desde nuestro punto de vista, no puede negarse que ciertas modalidades de la planificación territorial están cuestionadas en su consistencia teórica y en su factibilidad en el nuevo contexto. Por lo pronto, está claro que ningún territorio puede desarrollarse a espaldas de la globalización y que esta supone lógicas espaciales y transnacionales. Pero también es cierto que el territorio sigue siendo una variable relevante, que importa no solo a los actores sociales que lo habitan, sino también a los actores económicos, para los cuales está lejos de ser un mero "soporte". En la sección siguiente se reseñan algunas visiones que enfatizan precisamente la revalorización de lo territorial en la globalización (Bervejillo, 1994, p. 18).

Los últimos estudios de la Cepal, dejan claro que el proceso de internacionalización de los países de América Latina no ha sido el más apropiado y en lugar de generar condiciones de desarrollo se está lejos de una posición simétrica y competitiva dentro de las lógicas del sistema internacional y da lugar a una nueva dimensión entre lo local y lo glocal. Por lo tanto, la globalización no se puede encarar si un territorio no está organizado, preparado y dispuesto a establecer estrategias de inserción acordes con sus necesidades y expectativas. "La valorización de lo local en relación dialéctica con lo global, ha dado lugar incluso a extraños neologismos, como glocal, para expresar la pertenencia de los dos ámbitos espaciales al mismo campo relacional" (Moncayo, 2004, p. 15).

La internacionalización de los territorios no es un problema nuevo. Los geógrafos y economistas han dado explicaciones alusivas a la relación de los territorios internamente, entre lo que se conoce como relaciones interregionales o extraterritoriales (para el caso exterior), pero su aplicación sí es reciente debido a que su internacionalización no corresponde a una oferta social, económica o política reconocida ni demandada a escala internacional y mundial de los territorios, sino a condiciones económicas, de comercio exterior o a vínculos diplomáticos, como se presenta para el caso colombiano.

A pesar de que la Constitución colombiana sostiene en su artículo 287 que "[...] las entidades territoriales gozan de autonomía para la gestión de sus intereses, dentro de los límites de la constitución y la ley; y que en tal virtud tienen derecho a gobernarse por autoridades propias, ejercer las competencias que les correspondan, administrar los recursos 
y participar en las rentas nacionales", en ninguna de sus competencias, ya sean de carácter departamental, municipal o especial, se abre la posibilidad de crear una agenda de características internacionales que pueda, por algún canal, ser incluida entre los insumos básicos para una formulación de la política exterior ante el respectivo Ministerio. Esto implica una desarticulación básica entre los principios orientadores de la política exterior (paz y desarrollo) y la competencia primordial de las entidades territoriales (Ardila, Cardona \& Tickner, 2002, p. 18).

Si las entidades territoriales fronterizas pueden emprender acciones gubernamentales hacia el exterior para buscar opciones de desarrollo regional (diplomacia centrífuga entre regiones), el mismo derecho debe ser reconocido a las demás entidades territoriales. Esto lleva a plantear los siguientes cuestionamientos: ¡cómo pueden las entidades territoriales (regiones) formular una agenda de política exterior regional en este contexto? ¿cómo incluir sus demandas en esta materia al sector central?, preguntas cuya respuesta se encuentra en los determinantes del mismo proceso de internacionalización.

Para ello habrá que considerar que no es seguro que los gobiernos regionales de todas las zonas fronterizas latinoamericanas (particularmente en los países con modelo de Estado unitario) puedan jugar un papel semejante al que han jugado en Europa. Además, en el concepto de "región" se dan diferencias notables, tanto al interior de América Latina como en comparación con la Unión Europea. Es por ello que serían probablemente más pertinentes unas fórmulas mixtas que hicieran participar a los gobiernos regionales y locales, sobre la base de asociaciones territoriales transfronterizas, que podrían incluir también a actores de la sociedad civil (Observatorio Cooperación Descentralizada, 2008, p. 135).

La preocupación por internacionalizar los territorios puede entenderse como un problema de aceptación y reconocimiento, dada la necesidad que tienen de atraer actores que contribuyan a su desarrollo y progreso, en donde las regiones responden de manera convergente y divergente a escenarios relacionados con la entrada de inversión, sujetos foráneos o intereses bajo los cuales se puedan considerar o conjugar las necesidades del departamento y las subregiones con intereses extranjeros de manera recíproca y complementaria.

El territorio latinoamericano será homogéneo y controlable para el capital transnacional y sus procesos de internacionalización, pero al interior de él, sólo una parte será homogeneizada e integrada, mientras algunos de los estadios históricos donde los dejó algunas de las modernizaciones inconclusas y deformadas (Pradilla, 1995, p. 67).
La internacionalización de los territorios asimilados como regiones, no puede señalarse como un reducto o producto del desbarajuste de algún modelo o propuesta político-económica, sino que debe verse como una clara transformación de las relaciones internacionales entre los Estados constituidos de maneras unitarias o federadas frente a las concepciones que emergen en cuanto a la percepción de sus regiones a escala general, pero con alto contenido político de orden territorial.

Las regiones colombianas se enfrentan a una paradoja sin elementos de resolución. Por un lado, se les exige aumentar su competitividad como territorios, con el fin de hacer una inserción exitosa dentro del sistema internacional, especialmente en la dimensión económica de los procesos globales; por el otro, se les establecen una serie de limitantes jurídicas, políticas e institucionales que inhiben la posibilidad de que las regiones construyan y desarrollen una agenda exterior propia, en algunos casos, con rasgos de independencia de la agenda exterior del Estado central. De esta manera, las regiones quedan en un híbrido económico-político según el cual son agentes económicos exteriores, pero al mismo tiempo son agentes político-administrativos sin capacidad de acción exterior propia (Botero, 2010, p. 156).

Explicar la escalada regional como consecuencia del agotamiento de algún modelo, sería recaer en señalamientos impropios que entorpecerían la misma capacidad de los territorios para analizar su presente y su futuro, en virtud de su posición en todos los campos en los que asisten, en comparación con las regiones que les son cercanas o lejanas y respecto de la misma vinculación que tienen con el Estado, dado que la internacionalización obedece a intereses propios y no a un marco estrictamente estatal.

\section{Política pública de internacionalización y modelo de gestión departamental en Antioquia}

Un buen escenario para iniciar el proceso de internacionalización es el que propone cada unidad departamental como parte de su capacidad de gestión interna. El departamento de Antioquia requiere una mirada internacional - no vista como escenario ad hoc, sino como meta-, para contribuir a la internalización y pulsación positiva de las agendas internacionales como marco de verificación del estado y rumbo de la gestión gubernamental regional en diversos renglones.

Antioquia es una región rica en experiencias para la promoción del desarrollo económico y social, en el diseño de mecanismos institucionales para tal propósito y en proyectos de cooperación conjunta de los sectores público, privado y

$90<$ Universidad de San Buenaventura, Cali - Colombia 
comunitario. Sin embargo, para potenciar estas iniciativas se requiere de un mecanismo que los articule en torno a objetivos de largo plazo. Este es el sentido de un plan estratégico para Antioquia, proyecto que está en proceso de formulación (Londoño, 2000, p. 2).

El primer paso es concebir o señalar los parámetros que esta iniciativa engendraría -a lo que no escapa el campo político-, pues desde ella se han gestado los grandes cambios del país, los departamentos y las regiones. Por ende, centrar la discusión a sobre la base del origen de la propuesta, reduciría los patrones de exigencias de la internacionalización como un proceso de articulación completa. Es una tarea compleja que debe partir de algún punto, para concebir los caminos que se deben seguir para alcanzar la meta de ser un territorio internacional.

Existe, la necesidad de un diseño de políticas públicas y de un marco jurídico claro para la acción internacional de los gobiernos no centrales, en donde intervengan los tres ámbitos de gobierno. Mientras tanto, tendremos que esperar el transcurso de cierto tiempo para evaluar los resultados de las estrategias hasta ahora implementadas (Ruiz, 2009, pp. 272-273).

En segunda instancia, reconocer las condiciones actuales y futuras del departamento con el fin de establecer los aciertos y desaciertos que la iniciativa podría traer. A partir de ello, se haría un autodiagnóstico de la realidad para encarar un proceso de tal magnitud y mediante las consideraciones obtenidas, indicar los pasos que en materia de internacionalización se deben acoger con el propósito de hacer una evaluación plena de los procesos conducentes a la internacionalización.

La internacionalización de Medellín y la región, deberá construirse sobre cinco ejes principales: fomento y promoción de exportaciones de bienes y servicios, inversión extranjera directa, cooperación, marketing territorial y seguimiento a los acuerdos comerciales. Todos ellos atravesados por iniciativas y programas de corte institucional (Cámara de Comercio, 2009, p. 163).

Para diseñar una política de internacionalización del territorio antioqueño, deben resolverse inquietudes como las relacionadas con los enfoques de política regional que han circundado al departamento y las subregiones. Por ende, el análisis político del territorio podría hacerse desde la visión teórica de las políticas públicas de internacionalización y retomar la preeminencia de la planeación estratégica encaminada hacia ella.

El proceso de internacionalización es un evento sistémico que requiere la presencia y participación de todos, incluidas las redes o conexiones internacionales que van más allá de la presencia diplomática y empresarial. Asimismo, es necesaria una vocería permanente en diferentes latitudes que lleve consigo la consigna de que los territorios o regiones no solo parten de una unidad estatal, sino que también están compuesto por regiones, ciudades, localidades etc., no necesariamente inmersas en las corrientes globalizadoras, sino interesadas en jalonar este proceso por cuenta propia, como sucedió con respecto a la lides de la cooperación.

En los años noventa, la cooperación internacional se enfocó crecientemente en temas de desarrollo local. Esta tendencia se estancó debido a un número de factores interrelacionados. Por una parte, la globalización resaltó la importancia de las interconexiones mundiales y la relativa reducción del rol de los Estados nacionales. Por otra, trazó nuevas relaciones entre los procesos locales e internacionales (Rhi-Sausi, 2000, p. 5).

La internacionalización es, ante todo, un proceso de transformación de los territorios y regiones que advierten la importancia de integrarse entre sí y con la comunidad internacional, pero para lograrlo requieren asimilar las corrientes internacionales con la puesta en marcha de las consideraciones, convicciones y pensamientos provenientes del exterior. El grado de preferencia se suma al despliegue de una serie de recursos para internacionalizar los departamentos y las subregiones.

Partimos de una reflexión que podríamos denominar proyección de lo local en el ámbito internacional o, si se prefiere, de internacionalización de la dimensión local. Se trata de un fenómeno en pleno auge en nuestros días. Es la expresión de una tendencia profunda de evolución de las relaciones internacionales que refleja los cambios registrados en este campo, como resultado a la vez del declive de los Estados nacionales, especialmente en contextos de integración regional, como el europeo, la aceleración de la mundialización económica y de la nueva importancia del territorio y del desarrollo a escala local (Proyecto local, 2010, p. 30).

La internacionalización busca dar elementos para que las transformaciones de orden económico, social, político y cultural, afirmen la importancia y significancia que esta tiene para los territorios y cómo la definen para sí mismos, y la endogenizan hacia los demás, debido a que la relación con la convergencia es cada vez más lejana por cuanto siempre existirán diferencias estructurales e incluso cíclicas entre los territorios que no permiten centralizarlos y mucho menos compararlos.

La internacionalización busca dar respuesta al territorio antioqueńo en cuanto a la posibilidad de ser tenido como referencia en el contexto internacional y definir el grado de apetencia de la propuesta en las subregiones que lo 
componen. Es decir, debe tener en cuenta las condiciones estratégicas y políticas frente a la internacionalización y evaluarla con los demás sujetos activos del departamento $\mathrm{y}$ con las instancias institucionales (Figura 1).

Figura 1

Análisis meta de la internacionalización

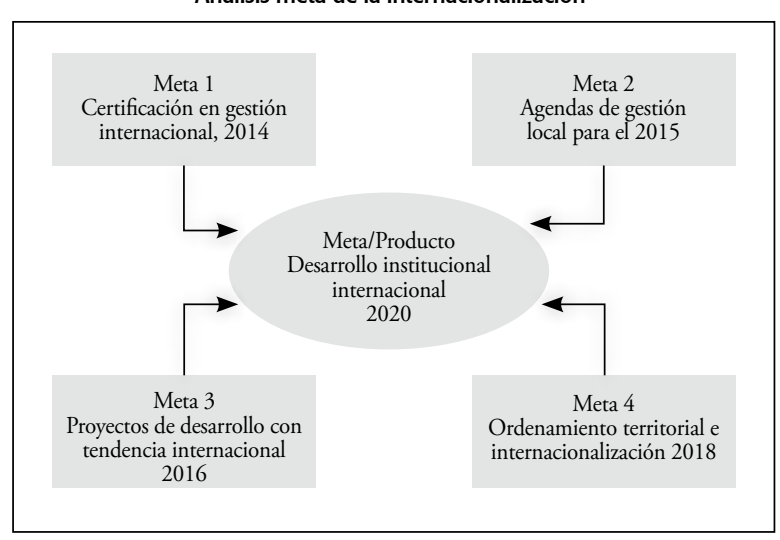

Fuente: Elaboración propia

Antioquia, constituido como uno de los departamentos de mayor referencia institucional cuando se trata de medir el grado de relación con el país en general, muestra ante el macromundo (el mundo) y el micromundo (quienes la conocen o saben de ella) la necesidad de construir caminos que le permitan encontrar su punto en el horizonte y ello lo ha intentado a partir de su definición como región; sin embargo, la mera determinación administrativa no es suficiente para internacionalizarse (Figura 2).

\section{Figura 2}

Análisis meso de la internacionalización

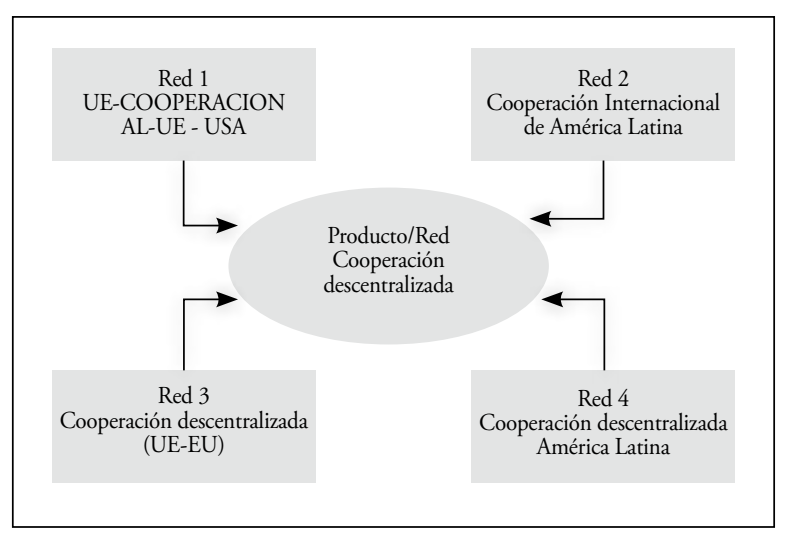

Fuente: Elaboración propia

Cabe advertir que por condiciones de espacialidad y territorio, los esfuerzos económicos, sociales y políticos se han centrado en la zona comprendida por el área metropolitana y particularmente por la capital y no propiamente en el departamento, hecho que obliga a mirar las posibilidades de internacionalización desde dos pers- pectivas: una geopolítica, que se enfoca en la subregión y una geoestratégica, relacionada con la capacidad de las subregiones para afrontar este proceso (Figura 3).

Figura 3

Análisis micro de la internacionalización

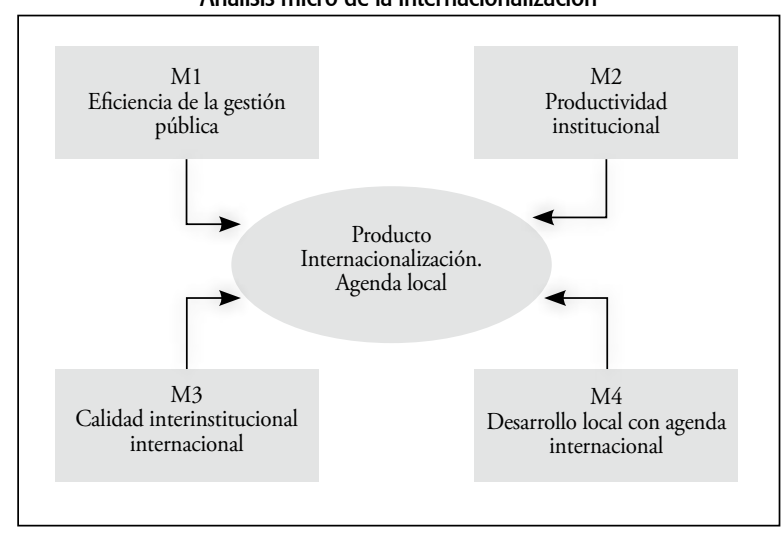

Fuente: Elaboración propia

$\mathrm{Al}$ momento de hacer balance de las perspectivas económicas y políticas, la internacionalización para los territorios queda en el vacío, puesto que aunque ambos enfoques han avanzado extraordinariamente en los últimos tiempos, aún son muchos los territorios que se encuentran fuera las corrientes de los procesos de internacionalización y de los desarrollos que tendrían que llevar a cabo para culminar con éxito esta faena.

Desde este escenario, se reitera el compromiso político de los distintos corporados para llevar a cabo con la mayor idoneidad e integralidad posibles los concursos convocados para disponer de las agendas de internacionalización como un paso adelante en la gestión, focalizada en la mirada terrenal de las relaciones. En este sentido, la concepción de los corporados con respecto a esta iniciativa, tendrá incidencia en la forma de hacer política y vincular las interacciones locales con la dimensión y exigencias internacionales.

\section{Discusión}

En el departamento de Antioquia, la internacionalización no se ha examinado con la profundidad que debiera, toda vez que aún no forma parte de la agenda gubernamental, a excepción de los dos últimos gobiernos, que si bien no han enfatizado los parámetros al respecto, han contribuido desde diversas perspectivas, como la cooperación internacional y la internacionalización.

La internacionalización tiene entre sus metas, resolver problemas o situaciones relacionadas con la imagen, la 
idea y la percepción que se tiene de algo o de alguien. En el caso particular de lo territorial, los fines pueden ser los mismos u otros relacionados con el interés primordial de difundir la imagen del territorio en todo el mundo; sin embargo, en ocasiones ello se hace sin una medida clara o por lo menos mensurable, lo cual obliga a considerar los aspectos relacionados con las capacidades del departamento para asumir dicha tarea.

Más que administradores se necesitan líderes; y más que dirigentes, creadores de una propuesta que prometa mejores condiciones de vida, dada la diversidad de opciones que Antioquia puede ofrecer en el contexto internacional. Sin embargo, para ello se requiere el concurso permanente de planificadores, investigadores y diseñadores de estrategias de desarrollo del departamento como un territorio de orden internacional, cuya capacidad no solo radica en la atención y prestación de servicios, sino también en la interacción simultánea como punto de acogimiento a extranjeros. Es fundamental que dentro de los planes de desarrollo se cuente con un ítem o acápite dedicado a las políticas y metas concebidas como elementos para la internacionalización de las mismas subregiones y sus zonas, a la par , claro está, con el departamento y la nación.

\section{Referencias}

Agudelo, J. (2011). Marco internacional de la cooperación para el desarrollo. En J. Agudelo (Autor), Variables sociopoliticas de la cooperación internacional para el desarrollo en América Latina y El Caribe (pp. 13-67). Bogota: Kimpres.

Ardila, M., Cardona, D., \& Tickner, A. B. (2002). El análisis de la política exterior colombiana: lugares comunes y grandes silencios. En M. Ardila, D. Cardona \& A. B. Tickner (Eds.), Prioridades y desafios de la politica exterior colombiana ( $\mathrm{pp}$. 17-35). Bogotá: FESCOL.

Proyecto local. (2010). Guía de orientaciones para la cooperación municipal al desarrollo. El poder de las ciudades y los gobiernos. Barcelona: Autor

Bervejillo, F. (1994). Territorios en la globalización. Cambio global y estrategias de desarrollo territorial. Recuperado de http://goo.gl/vzkTw3
Botero, M. H. (2010). La imposible misión de internacionalizar regiones en un estado centralista: El caso colombiano. Administración \& Desarrollo, 38, 145-158.

Cámara de Comercio. (2009). Plan regional de competitividad para Medellin, Valle de Aburrá y Antioquia. Medellín: Autor.

Cuervo, L. M. (2003). Pensar el territorio: los conceptos de ciudad-global y región en sus orígenes y evolución. Recuperado de http://goo.gl/rGZRqg

Devlin, R., \& Moguillansky, G. (2009). Alianzas público-privadas como estrategias nacionales de desarrollo a largo plazo. Revista Cepal 97. Recuperado de http://goo.gl/U9VnC0

Jiménez, W. G., Ochoa, A. M., \& Pineda, E. J. (2010). Internacionalización territorial, posibilidades y dificultades para los gobiernos subnacionales. Administración \& Desarrollo, $38,113-130$.

Londońo, Carlos A. (2000). La gestión del desarrollo en Antioquia, Colombia. Recuperado de http://goo.gl/EWchPy

Márquez, D., \& Pérez de Azpillaga, L. G. (2007). Otro mundo posible: cooperación con Iberoamérica interdisciplinar desde la Universidad de Sevilla. Cuadernos Geográficos 41, 205-224.

Moncayo, E. (2004). Las politicas regionales en Colombia. De la intervención activa al retraimiento del Estado. Bogotá: Universidad Externado de Colombia.

Observatorio de Cooperación Descentralizada Unión EuropeaAmérica Latina. (2008). Aportes de la cooperación descentralizada Unión Europea-América Latina a la cooperación territorial en América Latina. Elementos para el debate (Informe No. 1). Barcelona: Autor.

Osorio, D. (2010). ¿Internacionalización con o sin desarrollo local?: Un asunto de estrategia. Suma de negocios, 1(2), 73-89.

Pradilla, E. (1995). Los territorios latinoamericanos en la nueva fase de internacionalización neoliberal. Revista de Estudios Urbano Regionales, 21. Recuperado de http://goo.gl/Dj6ivW

Rhi-Sausi, J. (marzo, 2000). La cooperación internacional en los procesos de descentralización y regionalización de los países latinoamericanos. La experiencia Italia- Región de Atacama. Roma. Revista OIDLES, 2(3). Recuperado de http://goo.gl/dPqNsN

Ruiz, L. (2009). Las relaciones internacionales de los municipios. Convergencia. Revista de Ciencias Sociales, 16, 253-275. 